\title{
EXTENSIONS OF DIFFERENTIAL FIELDS. III
}

\author{
E. R. KOLCHIN
}

The purpose of the present note is to show how the point of view of a preceding paper ${ }^{1}$ can be used in developing the concepts of resolvent, dimension, and order introduced by J. F. Ritt in his theory of algebraic differential equations. ${ }^{2}$ The present development, in addition to being simpler in some instances, has the advantage of being valid for abstract differential fields as opposed to fields of meromorphic functions of a complex variable, as used by Ritt. I shall also take the opportunity to correct mistakes in a related paper. ${ }^{3}$ The notation and definitions used will be as in Extensions I and II.

1. Resolvents, dimension, and order. Let $\mathcal{F}$ be a differential field (ordinary or partial) of characteristic 0 , and let $y_{1}, \cdots, y_{n}$ be unknowns. If $\Pi$ is a prime differential ideal in $\mathcal{F}\left\{y_{1}, \cdots, y_{n}\right\}$ other than $\mathcal{F}\left\{y_{1}, \cdots, y_{n}\right\}$ itself then $\Pi$ has a generic solution $\eta_{1}, \cdots, \eta_{n}$.

If the degree of differential transcendency of $\mathcal{F}\left\langle\eta_{1}, \cdots, \eta_{n}\right\rangle$ over $\mathcal{F}$ is $q$ then $0 \leqq q<n$, and precisely $q$ of the elements $\eta_{1}, \cdots, \eta_{n}$ are differentially algebraically independent over $\mathcal{F}$. Suppose, say, that $\eta_{1} \cdots, \eta_{q}$ are independent in this way, that is, that $I I$ does not contain a nonzero differential polynomial in $y_{1}, \cdots, y_{q}$, but does in $y_{1}, \cdots, y_{q}, y_{j}$ for each $j>q$. In Ritt's terminology $y_{1} \cdots, y_{q}$ is a complete set of arbitrary unknowns for $\Pi$. It is natural to call $q$ the dimension of $\Pi$ (in symbols, $\operatorname{dim} \Pi$ ).

Suppose henceforth that $\mathcal{F}$ is ordinary. It is easy to see that the degree of transcendency of $\mathcal{F}\left\langle\eta_{1}, \cdots, \eta_{n}\right\rangle$ over $\mathcal{F}\left\langle\eta_{1}, \cdots, \eta_{q}\right\rangle$ (both these differential fields being considered as fields) is finite. We denote the degree of transcendency of any field $\mathcal{H C}$ over a subfield $G$ by $\partial^{0} \mathfrak{H C} / G$. It will be seen that it is natural to call the integer $\partial^{0} \mathcal{F}\left\langle\eta_{1}, \cdots, \eta_{n}\right\rangle / \mathcal{F}\left\langle\eta_{1}, \cdots, \eta_{q}\right\rangle$ the order of $\Pi$ with respect to $y_{1}, \cdots, y_{q}$ (when the set of arbitrary unknowns is understood, for example when $q=0$, we use the notation: ord II).

Presented to the Society, November 2, 1946; received by the editors October 10, 1946.

${ }^{1}$ Kolchin, Extensions of differential fields, I, Ann. of Math. vol. 43 (1942) pp. 724729. We shall refer to this paper as Extensions I.

2 The subject matter treated here, together with some of the material from $E x$ tensions I, is roughly parallel to the contents of $\$ \$ 24-31,75$ of Ritt, Differential equations from the algebraic standpoint, Amer. Math. Soc. Colloquium Publications, vol. 14, New York, 1932.

${ }^{3}$ Kolchin, Extensions of differential fields, II, Ann. of Math. vol. 45 (1944) pp. 358361. We shall refer to this paper as Extensions II. 
If $\mathcal{F}\left\langle\eta_{1}, \cdots, \eta_{q}\right\rangle$ contains a nonconstant (which is the case either when $\mathcal{F}$ does or when $q>0$ ) then by Extensions I there is an $\omega$ such that $\mathcal{F}\left\langle\eta_{1}, \cdots, \eta_{q}, \omega\right\rangle=\mathcal{F}\left\langle\eta_{1}, \cdots, \eta_{n}\right\rangle$. Let $A=A\left(\eta_{1}, \cdots, \eta_{q}, w\right)$ be an irreducible differential polynomial in $\mathcal{F}\left\langle\eta_{1}, \cdots, \eta_{q}\right\rangle\{w\}$, with solution $w=\omega$, of lowest possible order. Since $\omega$ and its first ord $\Pi$ derivatives must be algebraically dependent over $\mathcal{F}\left\langle\eta_{1}, \cdots, \eta_{q}\right\rangle$, the order of $A$ is not greater than ord $\Pi$. On the other hand, if the order of $A$ is $p$ then the $p$ th derivative (and consequently all the derivatives) of $\omega$ is algebraically dependent over $\mathcal{F}\left\langle\eta_{1}, \cdots, \eta_{q}\right\rangle$ on $\omega$ and its first $p-1$ derivatives, so that ord $\Pi=\partial^{0} \mathcal{F}\left\langle\eta_{1} \cdots, \eta_{n}\right\rangle / \mathcal{F}\left\langle\eta_{1}, \cdots, \eta_{q}\right\rangle$ $=\partial^{0} \mathcal{F}\left\langle\eta_{1}, \cdots, \eta_{q}, \omega\right\rangle / \mathcal{F}\left\langle\eta_{1}, \cdots, \eta_{q}\right\rangle \leqq p$. Therefore the order of $A$ in $w$ is $p=$ ord $\Pi$. $A\left(y_{1}, \cdots, y_{q}, w\right)$ is called a resolvent of $\Pi$. (Actually, this is a slight generalization of Ritt's resolvent, which must be in $\mathcal{F}\left\{y_{1}, \cdots, y_{q}, w\right\}$ instead of merely in $\left.\mathcal{F}\left\langle y_{1}, \cdots, y_{q}\right\rangle\{w\}.\right)$

Let $G$ be a differential extension field of $\mathcal{F}$, let $\{\Pi\}=\Pi_{1} \cap \cdots \cap \Pi_{r}$ be the decomposition into prime components (that is, prime differential ideals none of which contains another) of the perfect differential ideal generated by $\Pi$ in $G\left\{y_{1}, \cdots, y_{n}\right\}$, and let $A_{1}\left(y_{1}, \cdots, y_{q}, w\right) \cdots A_{s}\left(y_{1}, \cdots, y_{q}, w\right)$ be the complete factorization of $A\left(y_{1}, \ldots, y_{q}, w\right)$ in $G\left\langle y_{1}, \ldots, y_{q}\right\rangle\{w\}$. Each $A_{i}\left(y_{1}, \cdots, y_{q}, w\right)$ is of order $p$ in $w$, for a factor of $A\left(y_{1}, \cdots, y_{q}, w\right)$ of order less than $p$ would be a common factor of the coefficients in $A\left(y_{1}, \cdots, y_{q}, w\right)$ when $A\left(y_{1}, \cdots, y_{q}, w\right)$ is considered as a polynomial in $w_{p}$, the pth derivative of $w$. We shall now establish Ritt's result that $r=s$ and each $A_{i}\left(y_{1}, \cdots, y_{q}, w\right)$ is a resolvent of one $\Pi_{j}$. This result implies that $\Pi$ decomposes if and only if $A\left(y_{1}, \cdots, y_{q}, w\right)$ factors, and that each prime component in the decomposition has the same order as $\Pi$ has.

Let $\eta_{1}^{\prime}, \cdots, \eta_{n}{ }^{\prime}$ be a generic solution of $\Pi_{1}$. Then (by Extensions I, §1) $\eta_{1}^{\prime}, \cdots, \eta_{n}^{\prime}$ is a generic solution of $\Pi$, so that $\eta_{1}^{\prime} \rightarrow \eta_{1}, \cdots, \eta_{n}{ }^{\prime} \rightarrow \eta_{n}$ generates an isomorphism of $\mathcal{F}\left\langle\eta_{1}^{\prime}, \cdots, \eta_{n}^{\prime}\right\rangle$ onto $\mathcal{f}\left\langle\eta_{1}, \cdots, \eta_{n}\right\rangle$. Therefore if we let $\omega^{\prime}$ be the same differential rational function over $\mathcal{F}$ of $\eta_{1}^{\prime}, \cdots, \eta_{n}^{\prime}$ that $\omega$ is of $\eta_{1}, \cdots, \eta_{n}$, we shall have

$$
\mathcal{F}\left\langle\eta_{1}^{\prime}, \cdots, \eta_{\alpha}^{\prime}, \omega^{\prime}\right\rangle=\mathcal{F}\left\langle\eta_{1}^{\prime}, \cdots, \eta_{n}{ }^{\prime}\right\rangle \text {. }
$$

Now $\omega^{\prime}$ is a solution of $A^{\prime}=A\left(\eta_{1}^{\prime}, \cdots, \eta_{q}^{\prime}, w\right)$, and therefore of some $A_{i}^{\prime}=A_{i}\left(\eta_{1}^{\prime}, \cdots, \eta_{q}^{\prime}, w\right)$, say of $A_{1}^{\prime}$. Furthermore, $\omega^{\prime}$ is not a solution of two different $A_{1}^{\prime}$ 's, for $\omega^{\prime}$ does not annul the separant $\partial A^{\prime} / \partial w_{p}=\partial\left(A_{1}^{\prime} \cdots A_{s}^{\prime}\right) / \partial w_{p}$. Let $\omega^{\prime \prime}$ be a generic solution of the prime component of $\left\{A_{1}^{\prime}\right\}$ in $G\left\langle\eta_{1}^{\prime}, \cdots, \eta_{q}^{\prime}\right\rangle\{w\}$ not containing the separant $\partial A_{1}^{\prime} / \partial w_{p}$. Then $\omega^{\prime \prime}$ is a generic solution of the prime component of $\left\{A^{\prime}\right\}$ in $\mathcal{F}\left\langle\eta_{1}^{\prime}, \cdots, \eta_{q}^{\prime}\right\rangle\{w\}$ not containing the 
separant $\partial A^{\prime} / \partial w_{p}$, so that $\omega^{\prime \prime} \rightarrow \omega^{\prime}$ generates an isomorphism of $\mathcal{F}\left\langle\eta_{1}^{\prime}, \cdots, \eta_{q}^{\prime}, \omega^{\prime \prime}\right\rangle$ on to $\mathcal{F}\left\langle\eta_{1}^{\prime}, \cdots, \eta_{q}^{\prime}, \omega^{\prime}\right\rangle$, and a homomorphism of $G\left\langle\eta_{1}^{\prime}, \cdots, \eta_{q}^{\prime}\right\rangle\left\{\omega^{\prime \prime}\right\}$ onto $G\left\langle\eta_{1}^{\prime}, \cdots, \eta_{q}^{\prime}\right\rangle\left\{\omega^{\prime}\right\}$. Therefore, if for each $i>q$ we let $\eta_{i}^{\prime \prime}$ be the same differential rational function over $\mathcal{F}\left\langle\eta_{1}^{\prime}, \cdots, \eta_{q}^{\prime}\right\rangle$ of $\omega^{\prime \prime}$ as $\eta_{i}^{\prime}$ is of $\omega^{\prime}$, then $\eta_{1}^{\prime}, \cdots, \eta_{q}^{\prime}$, $\eta_{a+1}^{\prime \prime}, \cdots, \eta_{n}^{\prime \prime}$ is a generic solution of $\Pi$ and a solution of some $\Pi_{i}$. Since $\eta_{1}^{\prime}, \cdots, \eta_{n}^{\prime}$ must be a solution of the same $\Pi_{i}$, and since one $\Pi_{i}$ does not contain another, $\eta_{1}^{\prime}, \cdots, \eta_{q}^{\prime}, \eta_{q+1}^{\prime \prime}, \cdots, \eta_{n}^{\prime \prime}$ is a solution of $\Pi_{1}$, and indeed a generic one.

Therefore $\eta_{q+1}^{\prime \prime} \rightarrow \eta_{q+1}^{\prime}, \cdots, \eta_{n}^{\prime \prime} \rightarrow \eta_{n}^{\prime}$ generates an isomorphism of $G\left\langle\eta_{1}^{\prime}, \cdots, \eta_{q}^{\prime}, \eta_{q+1}^{\prime \prime}, \cdots, \eta_{n}^{\prime \prime}\right\rangle$ onto $G\left\langle\eta_{1}^{\prime}, \cdots, \eta_{n}^{\prime}\right\rangle, A_{1}^{\prime}$ is an irreducible differential polynomial in $G\left\langle\eta_{1}^{\prime}, \cdots, \eta_{q}^{\prime}\right\rangle\{w\}$, with solution $w=\omega^{\prime}$, of minimal degree, and $A_{1}\left(y_{1}, \cdots, y_{q}, w\right)$ is a resolvent of $\Pi_{1}$. In the same way, every $\Pi_{i}$ has an $A_{j}\left(y_{1}, \cdots, y_{q}, w\right)$ as a resolvent, so that $r \leqq s$. To show that there is no $A_{j}\left(y_{1}, \cdots, y_{q}, w\right)$ left over, for any $j$ let $\omega_{j}$ be a generic solution of the prime component of $\left\{A_{j}^{\prime}\right\}$ in $G\left\langle\eta_{1}^{\prime}, \cdots, \eta_{n}^{\prime}\right\rangle\{w\}$ not containing $\partial A_{j}^{\prime} / \partial w_{p}$. For each $i>q$ let $\eta_{j i}$ be the same differential rational function over $\mathcal{F}\left\langle\eta_{1}^{\prime}, \cdots, \eta_{q}^{\prime}\right\rangle$ of $\omega_{j}$ as $\eta_{i}^{\prime}$ is of $\omega^{\prime}$. Then $\eta_{1}^{\prime}, \cdots, \eta_{q}^{\prime}, \eta_{j, q+1}, \cdots, \eta_{j n}$ is a generic solution of $\Pi$ and therefore a solution of some $\Pi_{i}$, say $\Pi_{i_{0}}$. Therefore $\omega_{j}$ is a solution of the $A_{k}^{\prime}$ for which $A_{k}\left(y_{1}, \cdots, y_{q}, w\right)$ is a resolvent of $\Pi_{i_{0}}$. This implies that $A_{k}\left(y_{1}, \cdots, y_{q}, w\right)$ is divisible by $A_{j}\left(y_{1}, \cdots, y_{q}, w\right)$, so that $k=j$ and $A_{j}\left(y_{1}, \cdots, y_{q}, w\right)$ is a resolvent of a $\Pi_{i}$.

If $q=0$ and $\mathcal{F}$ consists solely of constants it is still true that each prime component of $\{\Pi\}$ has the same order as $\Pi$. To see this introduce a new unknown $u$ and let $\mathcal{F}^{\prime}=\mathcal{F}\langle u\rangle, G^{\prime}=G\langle u\rangle$. The perfect differential ideal generated by $\Pi$ in $\mathcal{F}^{\prime}\left\{y_{1}, \cdots, y_{n}\right\}$ is clearly prime and has the same order as $\Pi$ has. The prime components of the perfect differential ideal generated by $\Pi$ in $G^{\prime}\left\{y_{1}, \cdots, y_{n}\right\}$ are the perfect differential ideals generated by $\Pi_{1}, \cdots, \Pi_{r}$, and have the same order. Therefore ord $\Pi_{i}=$ ord $\Pi$ for each $i$.

2. Corrections to Extensions II. We refer now to the proof on page 359 of Extensions II. The derivation of the equation $\omega K(z)-H(z)$ $=\alpha A(z)$ is incorrect, for it rests on the unjustified assumption (see lines 18 and 17 from the bottom) that $\partial A(z) / \partial y_{p} \in G\{z\}$. To save the proof we delete in toto lines 22-4 from the bottom ("Denote the -..A(z):"), and replace them by the following considerations.

Let $\omega=H(y) / K(y)$ be any coefficient in $A(z)$ not merely an element of $\mathcal{F}$, with $H(y), K(y)$ free of common divisor. Clearly $\omega K(z)-H(z)$ $\in \Sigma$. 
Denote the lowest common denominator of the coefficients in $A(z)$ by $D(y)$, and let $B(y, z)=D(y) A(z)$. Then $B(y, z) \in \mathcal{F}\{y, z\}$, and $B(y, y)=0$. Since $A(z)$ is irreducible and one of the coefficients in $A(z)$ is unity, the irreducible factors of $B(y, z)$ are distinct and all have the same order in $z$ as $A(z)$ has.

Denoting the order of $B(y, z)$ in $y$ by $p$, let $B_{1}(y, z)$ be an irreducible factor of $B(y, z)$ of order $p$ in $y$. Let $\Lambda_{1}$ be the prime component of $\left\{B_{1}(y, z)\right\}$ which contains neither of the separants of $B_{1}(y, z)$. No other irreducible factor of $B(y, z)$ is in $\Lambda_{1}$, for such a factor would have the same order in $z$ as $B_{1}(y, z)$ and would be divisible by $B_{1}(y, z)$. Let $y, \zeta_{1}$ be a generic solution of $\Lambda_{1} . B(y, z) \in \Lambda_{1}$ but the separant of $B(y, z)$ with respect to $z$ is not in $\Lambda_{1}$ (for otherwise the separant of $B_{1}(y, z)$ would be in $\left.\Lambda_{1}\right)$. Therefore $\zeta_{1}$ is a nonsingular solution of $A(z)$, a solution of $\Sigma$, and a solution of $\omega K(z)-H(z)$. Thus $H(y) K(z)$ $-K(y) H(z)$ vanishes for the generic solution $y, \zeta_{1}$ of $\Lambda_{1}$, and is in $\Lambda_{1}$. With order in $y$ clearly not greater than $p, H(y) K(z)-K(y) H(z)$ must be divisible by $B_{1}(y, z)$.

Similarly, $H(y) K(z)-K(y) H(z)$ is divisible by all the irreducible factors $B_{1}(y, z), \cdots, B_{s}(y, z)$ of $B(y, z)$ which have order $p$ in $y$. Since all these $B_{i}(y, z)$ 's are distinct we may write

$$
H(y) K(z)-K(y) H(z)=L(y, z) B_{1}(y, z) \cdots B_{s}(y, z),
$$

where $L(y, z) \in \mathcal{F}\{y, z\}$. Moreover, if we denote the degree of $B(y, z)$ in $y_{p}$ (the pth derivative of $y$ ) by $d$, we see that the degree of $H(y) K(z)-K(y) H(z)$ in $y_{p}$ is not greater than $d$, that of $B_{1}(y, z)$ $\cdots B_{z}(y, z)$ is $d$, so that $L(y, z)$ is of degree 0 in $y_{p}$, that is, of order not greater than $p-1$ in $y$.

Let $B_{s+1}(y, z)$ be an irreducible factor of $B(y, z)$ of order $p-1$ in $y$, let $\Lambda_{s+1}$ be the prime component of $\left\{B_{s+1}(y, z)\right\}$ not containing the separants of $B_{s+1}(y, z)$, and let $y, \zeta_{s+1}$ be a generic solution of $\Lambda_{s+1}$. As with $y, \zeta_{1}$ before, we see that $y, \zeta_{s+1}$ is a solution of $H(y) K(z)$ $-K(y) H(z)$. But $y, \zeta_{s+1}$ is not a solution of any $B_{i}(y, z)$ with $i \leqq s$, for no such $B_{i}(y, z)$ is in $\Lambda_{s+1}$. Hence $y, \zeta_{s+1}$ is a solution of $L(y, z)$, and $L(y, z) \in \Lambda_{s+1}$. This implies, since the order of $L(y, z)$ in $y$ is not greater than $p-1$, that $L(y, z)$ is divisible by $B_{s+1}(y, z)$.

Similarly, $L(y, z)$ is divisible by all the irreducible factors $B_{s+1}(y, z)$, $\cdots, B_{t}(y, z)$ of order $p-1$ in $y$, so that

$$
H(y) K(z)-K(y) H(z)=M(y, z) B_{1}(y, z) \cdots B_{t}(y, z),
$$

where $M(y, z) \in \mathcal{F}\{y, z\}$. Moreover, if we denote the degree of $B(y, z)$ in $y_{p}, y_{p-1}$ by $e$, we see that the degree of $H(y) K(z)-K(y) H(z)$ in $y_{p}, y_{p-1}$ is not greater than $e$, that of $B_{1}(y, z) \cdots B_{t}(y, z)$ is $e$, 
so that $M(y, z)$ is of degree 0 in $y_{p}, y_{p-1}$, that is, of order not greater than $p-2$ in $y$.

Continuing in this way we finally arrive at an equation

$$
H(y) K(z)-K(y) H(z)=P(z) B_{1}(y, z) \cdots B_{w}(y, z),
$$

where $B_{1}(y, z), \cdots, B_{w}(y, z)$ are all the irreducible factors of $B(y, z)$. Since $H(z), K(z)$ have no common divisor, $H(y) K(z)-K(y) H(z)$ has no factor free of $y$ that is not also free of $z$. Therefore $P(z) \in \mathcal{F}$, and $H(y) K(z)-K(y) H(z)=a B(y, z)$, where $a \in \mathcal{F}$. The desired equation $\omega K(z)-H(z)=\alpha A(z)$ immediately follows.

The rest of the proof of the theorem as given in Extensions II is apparently correct.

Of the two examples given in Extensions II, the proof for Example 2 is incorrect, and I do not yet know whether that example is valid.

Columbia University 